\title{
Stability of Solitary Waves of a Fifth-Order Water Wave Model
}

\author{
Steve Levandosky*
}

\begin{abstract}
We consider the stability of solitary waves of a class of 5th order KdV equations. It is known that their stability is determined by the second derivative of a function of the wave speed $d(c)$. We perform a detailed investigation of the properties of this function, both analytically and numerically. For a class of homogeneous nonlinearities, we precisely determine the regions of wave speeds for which the solitary waves are stable or unstable.
\end{abstract}

Keywords: Water wave model; Solitary waves; Stability

\section{Introduction}

In this paper, we study the stability of solitary wave solutions of the fifth-order KdV equation

$$
u_{t}+u_{x x x x x}+b u_{x x x}=\left(f\left(u, u_{x}, u_{x x}\right)\right)_{x},
$$

where the nonlinearity has the variational form

$$
f(q, r, s)=F_{q}(q, r)-r F_{q r}(q, r)-s F_{r r}(q, r)
$$

for some $C^{2}$ function $F(q, r)$ that is homogeneous of degree $p+1$ for some $p>2$. Such equations have been studied by many authors as long-wave approximations to the waterwave equations. See, for example, Benney [2] and Olver [13] for the nonlinearity $F(u)=$ $-u^{3}$, Zufiria [14] and Hunter \& Scheurle [9] for the nonlinearity $F\left(u, u_{x}\right)=-u u_{x}^{2}$, and Craig \& Groves [6] for the nonlinearity $F\left(u, u_{x}\right)=u u_{x}^{2}-u^{3}$. Traveling wave solutions $u(x, t)=\varphi(x+c t)$ of equation (1.1) are described by the stationary equation

$$
\varphi_{x x x x}+b \varphi_{x x}+c \varphi=f\left(\varphi, \varphi_{x}, \varphi_{x x}\right) .
$$

Existence of solutions of this equation was considered by the author in [11]. Using the concentration-compactness principle of Lions [12], it was shown that minimizers of the functional

$$
I(u)=\frac{1}{2} \int\left(u_{x x}\right)^{2}-b\left(u_{x}\right)^{2}+c u^{2} d x
$$

*Mathematics and Computer Science Department, College of the Holy Cross, Worcester, MA 01610, spl@mathcs.holycross.edu 
subject to the constraint

$$
K(u)=\int F\left(u, u_{x}\right) d x=\lambda>0
$$

exist in the space $H^{2}(\mathbf{R})$, provided $c>0$ and $b<2 \sqrt{c}$. After appropriate re-scaling, these minimizers are solutions of the solitary wave equation (1.2). We shall refer to such solutions as ground states, and denote by $\mathcal{G}(b, c)$ the set of all ground states. Given a ground state $\varphi \in \mathcal{G}(b, c)$, we will denote its orbit by $\Omega_{\varphi}=\{\varphi(\cdot-y): y \in \mathbf{R}\}$. We remark here that uniqueness of ground state solutions up to translation would imply that $\mathcal{G}(b, c)=\Omega_{\varphi}$. At this time however, it is not known whether or not ground states are unique.

In this paper we are concerned with stability of either $\mathcal{G}(b, c)$ or $\Omega_{\varphi}$ in the $H^{2}$ norm. In the results that follow, we will use the following definition.

Definition 1.1. We say that a set $\mathcal{S}$ is stable with respect to the equation (1.1) if given $\epsilon>0$ there exists some $\delta>0$ such that if $g$ satisfies $\inf _{\psi \in \mathcal{S}}\|g-\psi\|_{H^{2}}<\delta$ then the solution $u(t)$ of (1.1) with initial data $u(0)=g$ exists for all $t>0$ and satisfies $\sup _{t \in[0, \infty)} \inf _{\psi \in \mathcal{S}} \| u(t)-$ $\psi \|_{H^{2}}<\epsilon$. Otherwise we say $\mathcal{S}$ is unstable with respect to (1.1).

Central to the stability analysis are the conserved quantities

$$
E(u)=\int \frac{1}{2}\left(u_{x x}\right)^{2}-\frac{b}{2}\left(u_{x}\right)^{2}-F\left(u, u_{x}\right) d x
$$

and

$$
Q(u)=\int \frac{1}{2} u^{2} d x
$$

which are interpreted as energy and momentum, respectively. Employing the framework developed by Grillakis, Shatah and Strauss [10] as applied in the case of the KdV equation by Bona, Souganidis and Strauss [3], it was shown in [11] that the stability of traveling waves in the space $H^{2}(\mathbf{R})$ is determined by the function

$$
d(c)=E(\varphi)+c Q(\varphi)
$$

where $\varphi$ is a traveling wave with speed $c$. More precisely, the main result is the following.

Theorem 1.2. Suppose $c>0$ and $b<2 \sqrt{c}$.

1. If $d^{\prime \prime}(c)>0$ then $\mathcal{G}(b, c)$ is stable.

2. If $d^{\prime \prime}(c)<0$ and $\varphi \in \mathcal{G}(b, c)$, then $\Omega_{\varphi}$ is unstable.

The hypothesis $d^{\prime \prime}(c)>0$ may be weakened to strict convexity of $d$ in some interval containing $c$.

In this paper we will be concerned with nonlinearities $F\left(u, u_{x}\right)$ that are homogeneous in both $u$ and $u_{x}$. That is, $F$ must satisfy

$$
F(\mu q, r)=\mu^{\alpha} F(q, r), \quad F(q, \mu r)=\mu^{\beta} F(q, r)
$$


for all $\mu \geq 0$, where $\alpha$ and $\beta$ are nonnegative and $\alpha+\beta=p+1>2$. In the special case $b=0$ the scaling properties of equation (1.2) lead to the explicit formula

$$
d(c)=d(1) c^{\gamma}, \quad \gamma=\frac{3 p-2 \beta+5}{4(p-1)}
$$

and it follows from Theorem 1.2 that we have stability for $p+2 \beta<9$ (since $\gamma>1$ ) and instability for $p+2 \beta>9$ (since $\gamma<1$ ). However, for $b \neq 0$ there is no explicit formula for $d$, so we cannot easily apply Theorem 1.2. This situation was improved upon by Angulo Pava [1] using ideas from Gonçalves Ribeiro [8]. The main result of [1] is the following instability theorem.

Theorem 1.3. (Angulo Pava) $\Omega_{\varphi}$ is unstable if

$$
\begin{aligned}
& \text { 1. } b=0 \text { and } p+2 \beta>9 \text {, or } \\
& \text { 2. } b<0 \text { and } p+2 \beta \geq 9 \text {, or } \\
& \text { 3. } b>0, b \text { small, and } p+2 \beta>9
\end{aligned}
$$

By different methods that make use of the Evans function, Bridges and Derks [4] proved linear instability in the case of $b=0$ and $p+2 \beta>9$.

The purpose of this paper is to present a detailed investigation of the properties of $d$, both analytically and numerically. The main result is that, for several nonlinearities of the type described above, we are able to completely determine the values of the parameter $b$ and wave speed $c$ for which the ground states are stable and unstable. See Table 1.

The paper is organized as follows. In section 2 we summarize the known results about existence and non-existence of ground states and their variational properties. In section 3 we use the homogeneity of the nonlinearity to establish a scaling property of $d$. We use this identity to reduce the problem of computing $d^{\prime \prime}$ at all points in the $(b, c)$ plane to computing $d^{\prime \prime}$ along two line segments. Case 3 of Theorem 1.3 is a corollary of this calculation. We also obtain bounds on $d$ near the boundary of its domain that imply convexity of $d$. Finally, in Section 4 we outline the numerical method employed to compute solutions of the solitary wave equation (1.2), and explain how these computations allow us to determine the sign of $d^{\prime \prime}$ for all $b$ and $c$. Results for several different nonlinearities are presented.

\section{Solitary Waves}

In this section we will summarize the known results about existence of solitary wave solutions of equation (1.1). We first observe that the eigenvalues of the system obtained by linearizing the solitary wave equation (1.2) about the zero solution are given by

$$
\lambda= \pm \sqrt{\frac{-b \pm \sqrt{b^{2}-4 c}}{2}} .
$$

Figure 1 shows the possible configurations of eigenvalues. For $c>0$ and $b>2 \sqrt{c}$ the eigen- 


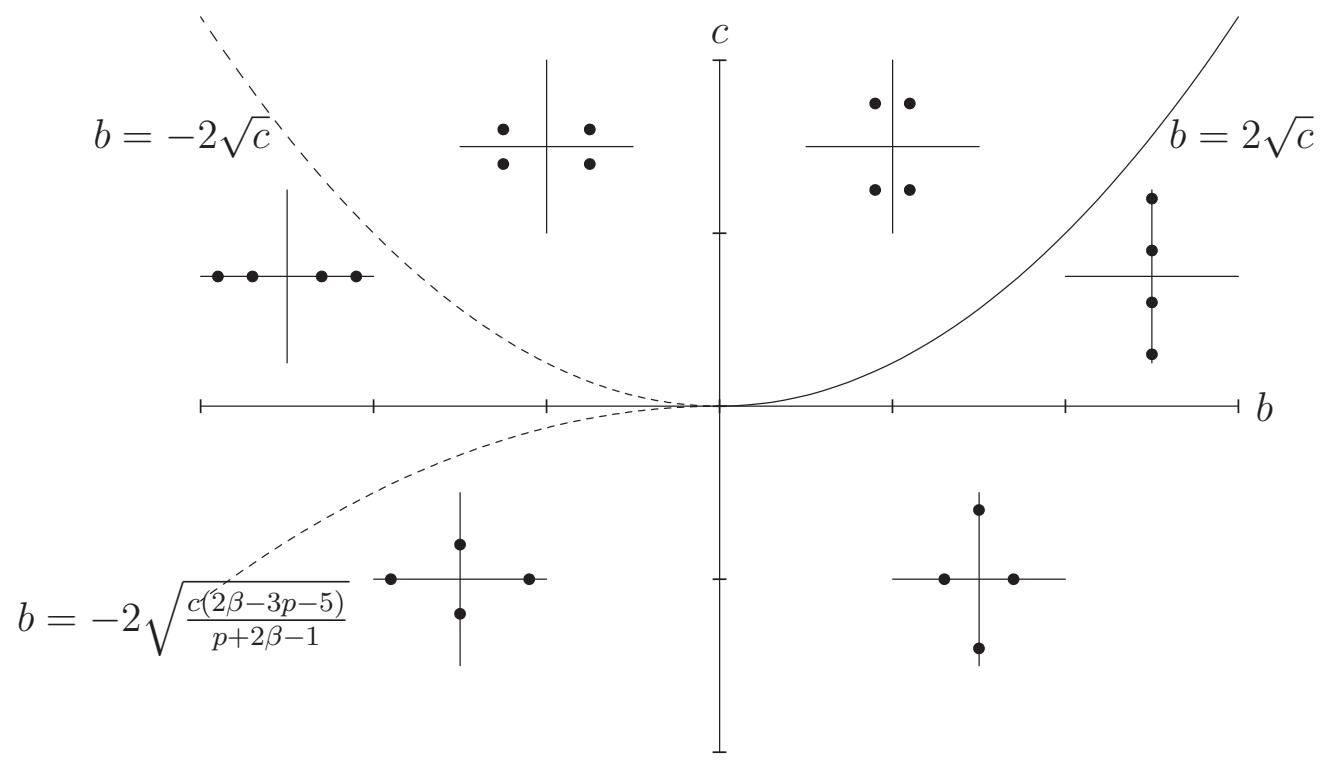

Figure 1: Eigenvalues for the linearized solitary wave equation.

values are purely imaginary, so $H^{2}$ solitary waves only exist under very special conditions on the nonlinear term. As mentioned above, for $c>0$ and $b<2 \sqrt{c}$ there do exist solitary waves in $H^{2}$, and these are the solutions to which the above stability theorems apply. The dashed curve $b=-2 \sqrt{c}$ indicates the transition from solitary waves with exponentially decaying oscillatory tails to solitary waves with exponentially decaying, but non-oscillatory tails. See Figure 2. For $c<0$ we have non-existence in the region to the right of the curve $b=-2 \sqrt{\frac{c(2 \beta-3 p-5)}{p+2 \beta-1}}$ in Figure 1. The proof of this fact requires the following Pohozaev-type identity.

Lemma 2.1. Let $\varphi$ be a solution of $(1.2)$ in $H^{2}(\mathbf{R})$. Then

$$
\int \frac{3}{2}\left(\varphi_{x x}\right)^{2}-\frac{b}{2}\left(\varphi_{x}\right)^{2}-\frac{c}{2} \varphi^{2} d x=(\beta-1) \int F\left(\varphi, \varphi_{x}\right) .
$$

Proof. Multiplying equation (1.2) by $\varphi_{x}$ and integrating gives

$$
\begin{aligned}
\varphi_{x x x} \varphi_{x}-\frac{1}{2} \varphi_{x x}^{2}+\frac{b}{2} \varphi_{x}^{2}+\frac{c}{2} \varphi^{2} & =\int f\left(\varphi, \varphi_{x}, \varphi_{x x}\right) \varphi_{x} d x \\
& =\int F_{q}\left(\varphi, \varphi_{x}\right) \varphi_{x}-\left(F_{r}\left(\varphi, \varphi_{x}\right)\right)_{x} \varphi_{x} d x \\
& =-F_{r}\left(\varphi, \varphi_{x}\right) \varphi_{x}+\int F_{q}\left(\varphi, \varphi_{x}\right) \varphi_{x}+F_{r}\left(\varphi, \varphi_{x}\right) \varphi_{x x} d x \\
& =-F_{r}\left(\varphi, \varphi_{x}\right) \varphi_{x}-\int F\left(\varphi, \varphi_{x}\right)_{x} d x \\
& =-\beta F\left(\varphi, \varphi_{x}\right)+F\left(\varphi, \varphi_{x}\right) \\
& =(1-\beta) F\left(\varphi, \varphi_{x}\right)
\end{aligned}
$$



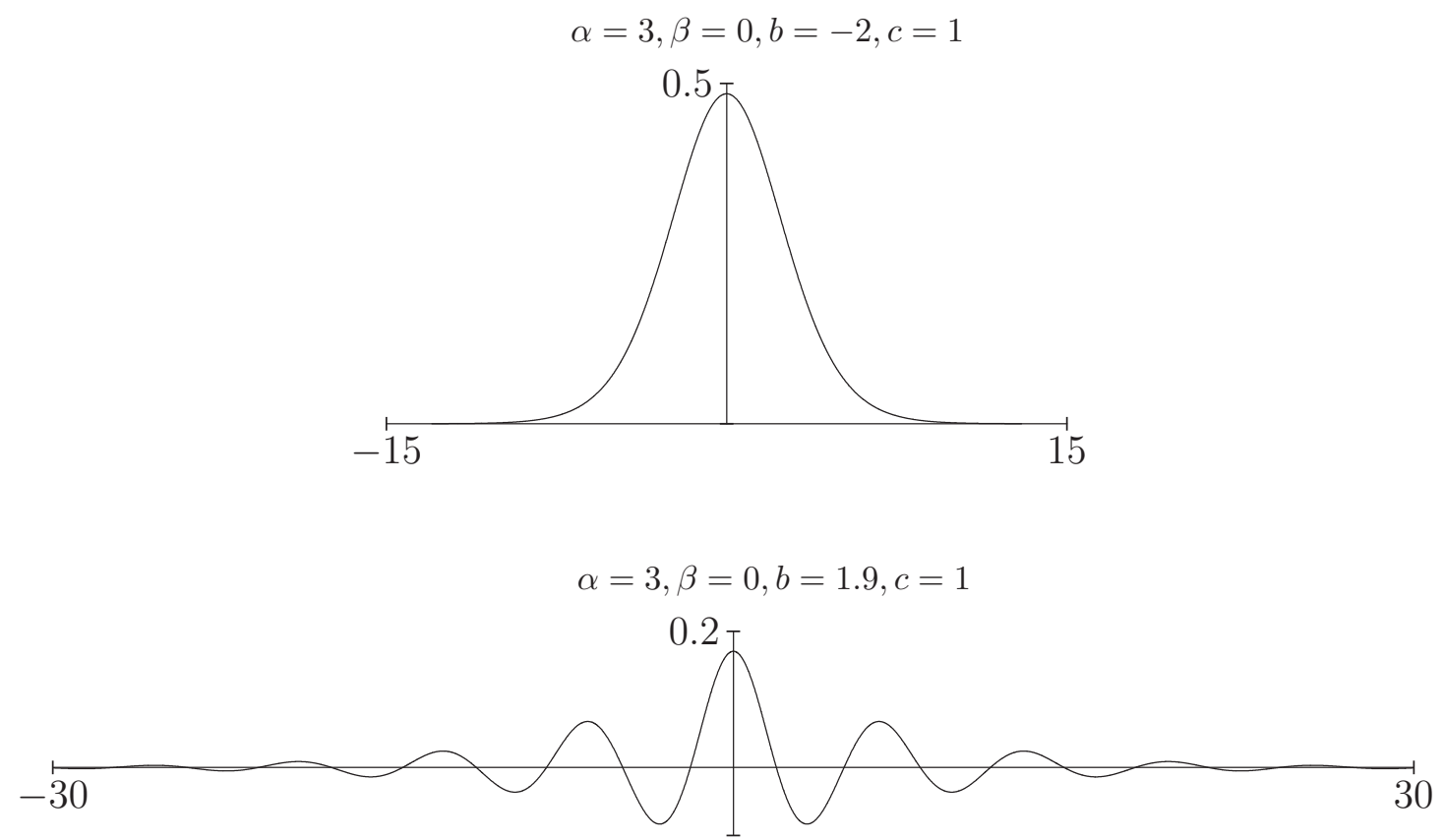

Figure 2: Examples of solitary waves.

where in the second to last line we have made use of the fact that $r F_{r}=\beta F$ by (1.3). The lemma then follows by integrating over $\mathbf{R}$.

Lemma 2.2. Suppose $c<0$ and $b>-2 \sqrt{\frac{c(2 \beta-3 p-5)}{p+2 \beta-1}}$. Then equation (1.2) has no nontrivial solutions in $H^{2}(\mathbf{R})$.

Proof. Suppose $\varphi$ is an $H^{2}$ solution of (1.2). Multiplying (1.2) by $\varphi$ and integrating gives

$$
2 I(\varphi)=(p+1) K(\varphi) .
$$

Together with equation (2.1) this implies

$$
\left(\beta-1-\frac{3(p+1)}{2}\right) \int \varphi_{x x}^{2} d x-b\left(\beta-1+\frac{p+1}{2}\right) \int \varphi_{x}^{2} d x+c\left(\beta-1+\frac{p+1}{2}\right) \int \varphi^{2} d x=0 .
$$

Since $c<0,0 \leq \beta \leq p+1$ and $p>1$, the coefficients of $\int \varphi_{x x}^{2}$ and $\int \varphi^{2}$ are both negative. Thus a nontrivial solution is impossible if either $b>0$ or

$$
b^{2}\left(\beta-1+\frac{p+1}{2}\right)^{2}<4\left(\beta-1-\frac{3(p+1)}{2}\right) c\left(\beta-1+\frac{p+1}{2}\right) .
$$

The inequality reduces to

$$
b^{2}<\frac{4 c(2 \beta-3 p-5)}{p+2 \beta-1}
$$

from which the lemma follows. 
In the case of the pure power nonlinearity $F(u)=u^{\alpha}$, it was shown by Dey, Khare and Kumar [7] that there are exact solitary wave solutions. In particular, for $c>0$ and $b / \sqrt{c}=-\left(\frac{\alpha}{2}+\frac{2}{\alpha}\right)$, we have

$$
\varphi(x)=\left(\frac{c(\alpha+2)(3 \alpha-2)}{8 \alpha^{2}}\right)^{\frac{1}{\alpha-2}} \cdot \operatorname{sech}^{\frac{4}{\alpha-2}}\left(\frac{(\alpha-2) x \sqrt{\left(-\frac{c}{b}\right)\left(\alpha^{2}+4\right)}}{4 \alpha}\right)
$$

In Figure 1 these solitary waves lie along a parabolic arc to the left of the dashed curve $b=-2 \sqrt{c}$.

\section{Properties of the Function $d$}

In this section we consider the function $d(c)$ that determines the stability of ground state solitary waves. The main result is a scaling identity which plays a central role in the numerical computation of $d$. We also derive bounds on $d$ near the boundary of its domain and use them to conclude that there must be intervals of stable solitary waves for certain nonlinear terms.

First, recall that for $c>0$ and $b<2 \sqrt{c}$ we denote by $\mathcal{G}(b, c)$ the set of ground state solutions of (1.2) obtained by minimizing

$$
I(u) \equiv I(u ; b, c)=\frac{1}{2} \int\left(u_{x x}\right)^{2}-b\left(u_{x}\right)^{2}+c u^{2} d x
$$

subject to the constraint

$$
K(u)=\int F\left(u, u_{x}\right) d x=\lambda>0
$$

and then rescaling appropriately. Since $K$ is homogeneous of degree $p+1$, these ground states achieve the minimum

$$
m(b, c)=\inf _{u \in H^{2}, K(u)>0} \frac{I(u ; b, c)}{K(u)^{2 / p+1}} .
$$

Applying equation (2.2), this becomes

$$
m(b, c)=\frac{p+1}{2} K(\varphi)^{\frac{p-1}{p+1}}
$$

and therefore by the definition of $d$ in equation (1.4) and the fact that $E(u)+c Q(u)=$ $I(u)-K(u)$ for all $u \in H^{2}(\mathbf{R})$, we have

$$
d(b, c)=I(\varphi ; b, c)-K(\varphi)=\frac{p-1}{2} K(\varphi)=\frac{p-1}{2}\left(\frac{2 m(b, c)}{p+1}\right)^{\frac{p+1}{p-1}} .
$$

This proves that $d$ is well-defined, and that one may study $d$ by considering the Rayleigh quotient that defines $m$. Using such an approach one may obtain the regularity properties of stated in the following lemma. For the dependence on the variable $c$, this was proved in [11] (Lemma 3.1 and Lemma 3.2). The proof of the results concerning the dependence on $b$ is identical. 
Lemma 3.1. The function $d$ is continuous, strictly increasing in $c$ and strictly decreasing in $b$ on the domain $c>0, b<2 \sqrt{c}$. For each fixed $b$ the partial derivative $\partial d / \partial c(b, c)$ exists for all but countably many $c$, and for each fixed $c$ the partial derivative $\partial d / \partial b(b, c)$ exists for all but countably many $b$. At points of differentiability, we have

$$
\begin{aligned}
& \frac{\partial d}{\partial c}=\frac{1}{2} \int \varphi^{2} d x \\
& \frac{\partial d}{\partial b}=-\frac{1}{2} \int\left(\varphi_{x}\right)^{2} d x
\end{aligned}
$$

where $\varphi$ is any element of $\mathcal{G}(b, c)$.

Since we need to consider higher derivatives of $d$, we make the following assumption.

Assumption 3.2. The function $d$ is $C^{2}$ in the region $c>0, b<2 \sqrt{c}$.

We now state the key scaling property of the function $m(b, c)$.

Lemma 3.3. Suppose $c>0$ and $b<2 \sqrt{c}$. Then for any $s>0$,

$$
m\left(s b, s^{2} c\right)=s^{\frac{3 p-2 \beta+5}{2(p+1)}} m(b, c) .
$$

Proof. We actually prove a slightly more general result. For $a>0$ and $c>0$, define

$$
m(a, b, c)=\inf _{u \in H^{2}, K(u)>0} \frac{I(u ; a, b, c)}{K(u)^{2 / p+1}},
$$

where

$$
I(u ; a, b, c)=\frac{1}{2} \int a\left(u_{x x}\right)^{2}-b\left(u_{x}\right)^{2}+c u^{2} d x
$$

Then, for $b<2 \sqrt{a c}$ and any $\lambda>0$ and $\mu>0$ we have

$$
m\left(\lambda \mu^{4} a, \lambda \mu^{2} b, \lambda c\right)=\lambda \mu^{\frac{p+2 \beta-1}{p+1}} m(a, b, c)
$$

The lemma follows by choosing $a=1, \lambda=s^{2}$ and $\mu=s^{-1 / 2}$. To prove (3.2), first note that, for any $u \in H^{2}$ and $\lambda>0$,

$$
I(u ; \lambda a, \lambda b, \lambda c)=\lambda I(u ; a, b, c)
$$

and thus $m(\lambda a, \lambda b, \lambda c)=\lambda m(a, b, c)$. Next, given $u \in H^{2}$, let $v(x)=u(\mu x)$. Then

$$
I(v ; a, b, c)=\frac{1}{2} \mu^{-1} \int \mu^{4} a\left(u_{x x}\right)^{2}-\mu^{2} b\left(u_{x}\right)^{2}+c u^{2} d x=\frac{1}{\mu} I\left(u ; \mu^{4} a, \mu^{2} b, c\right)
$$

and

SO

$$
K(v)=\frac{1}{\mu} \int u^{\alpha} \mu^{\beta} u_{x}^{\beta} d x=\mu^{\beta-1} K(u)
$$

$$
\frac{I(v ; a, b, c)}{K(v)^{2 / p+1}}=\mu^{-\frac{p+2 \beta-1}{p+1}} \frac{I\left(u ; \mu^{4} a, \mu^{2} b, c\right)}{K(u)^{2 / p+1}}
$$

and thus

$$
m\left(\mu^{4} a, \mu^{2} b, c\right)=\mu^{\frac{p+2 \beta-1}{p+1}} m(a, b, c) .
$$


By the relationship between $d$ and $m$ given by (3.1), we have the following scaling property of $d$.

Corollary 3.4. Suppose $c>0$ and $b<2 \sqrt{c}$. Then for any $s>0$,

$$
d\left(s b, s^{2} c\right)=s^{\frac{3 p-2 \beta+5}{2(p-1)}} d(b, c) .
$$

By the scaling property (3.3), it follows that the values of $d(b, c)$ along any curve of the form $b / \sqrt{c}=k$ for some constant $k$ are determined by the value of $d$ at any one point on the curve. It therefore suffices to compute $d$ along the line segments $\Gamma_{1}=\{(b, 1):-1 \leq b<2\}$ and $\Gamma_{2}=\{(-1, c): 0<c \leq 1\}$, since every curve of the form $b / \sqrt{c}=k<2$ intersects of these segments. See Figure 3. The derivatives of $d$ along these paths are calculated as

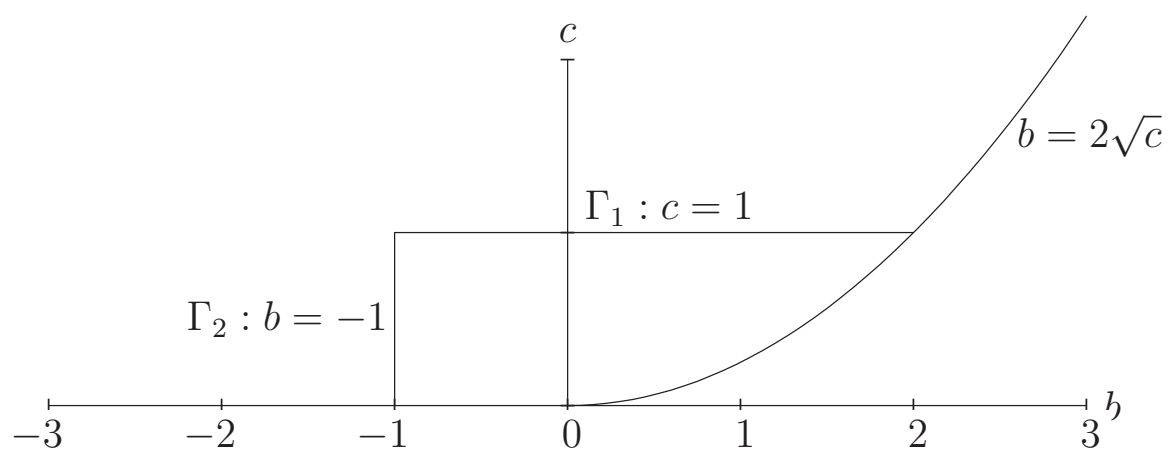

Figure 3: The segments $\Gamma_{1}$ and $\Gamma_{2}$.

follows.

Along $\Gamma_{1}$ : First rewrite the scaling property in the form

$$
d(b, c)=c^{\gamma} d\left(\frac{b}{\sqrt{c}}, 1\right), \quad \gamma=\frac{3 p-2 \beta+5}{4(p-1)} .
$$

Then differentiate with respect to $c$ to find

$$
d_{c}(b, c)=\gamma c^{\gamma-1} d\left(\frac{b}{\sqrt{c}}, 1\right)-\frac{1}{2} b c^{\gamma-\frac{3}{2}} d_{b}\left(\frac{b}{\sqrt{c}}, 1\right)
$$

and

$$
\begin{aligned}
d_{c c}(b, c) & =\gamma(\gamma-1) c^{\gamma-2} d\left(\frac{b}{\sqrt{c}}, 1\right)-\frac{1}{2} b\left(2 \gamma-\frac{3}{2}\right) c^{\gamma-\frac{5}{2}} d_{b}\left(\frac{b}{\sqrt{c}}, 1\right)+\frac{1}{4} b^{2} c^{\gamma-3} d_{b b}\left(\frac{b}{\sqrt{c}}, 1\right) \\
& =c^{\gamma-2}\left[\gamma(\gamma-1) d(r, 1)-\frac{1}{2} r\left(2 \gamma-\frac{3}{2}\right) d_{b}(r, 1)+\frac{1}{4} r^{2} d_{b b}(r, 1)\right] .
\end{aligned}
$$

where $r=b / \sqrt{c}$.

Along $\Gamma_{2}$ : For $b<0$, the scaling relation may be rewritten

$$
d(b, c)=(-b)^{2 \gamma} d\left(-1, c / b^{2}\right), \quad \gamma=\frac{3 p-2 \beta+5}{4(p-1)},
$$

so differentiation with respect to $c$ gives

$$
d_{c c}(b, c)=(-b)^{2 \gamma-4} d_{c c}\left(-1, c / b^{2}\right) .
$$


Thus, once the values of $d$ are known along $\Gamma_{1}$ and $\Gamma_{2}$, equations (3.4) and (3.5) may be used to find $d_{c c}$ at any point $(b, c)$ in the domain of $d$. Furthermore, from (3.4), we have the following result for small $b$.

Lemma 3.5. Suppose Assumption 3.2 holds.

(a) Suppose $p+2 \beta<10$. Then for $c>0$ and $b$ sufficiently small, the set of ground states $\mathcal{G}(b, c)$ is stable.

(b) Suppose $p+2 \beta>10$. Then for $c>0$ and $b$ sufficiently small, the set of ground states $\mathcal{G}(b, c)$ is unstable.

Proof. By (3.4) and Assumption 3.2, for fixed $c>0$,

$$
\lim _{b \rightarrow 0} d_{c c}(b, c)=\gamma(\gamma-1) c^{\gamma-2} d(0,1) .
$$

The lemma then follows since $\gamma>1$ when $p+2 \beta<10$ and $0<\gamma<1$ when $p+2 \beta>10$.

For the remainder of this paper we will focus on two classes of nonlinearities. We will consider even nonlinearities of the form $F\left(u, u_{x}\right)=|u|^{\alpha}\left|u_{x}\right|^{\beta}$ and odd nonlinearities $F\left(u, u_{x}\right)= \pm \operatorname{sign}(u)|u|^{\alpha}\left|u_{x}\right|^{\beta}$. This grouping appears to be natural given the behavior of the function $d$ near the boundary curve $c=b^{2} / 4$. This also permits us to consider arbitrary (non-integer) values of $\alpha$ and $\beta$. In our numerical computations, however, we restrict attention to integral $\alpha$ and $\beta$.

The following results give useful bounds on $d$ near the boundaries of its domain; $c=0$ for $b<0$ and $c=b^{2}$ for $b>0$. The idea is to patch together two solutions of the linear equation obtained by dropping the nonlinear term in the solitary wave equation in such a way as to construct a test function for the Rayleigh quotient that defines $m(b, c)$ and use the equation relating $d$ and $m$ to obtain an estimate on $d$. The estimate near $c=0$ applies to both the evan and odd nonlinearities.

Lemma 3.6. Let $F\left(u, u_{x}\right)=|u|^{\alpha}\left|u_{x}\right|^{\beta}$ or $F\left(u, u_{x}\right)=\operatorname{sign}(u)|u|^{\alpha}\left|u_{x}\right|^{\beta}$, where $\alpha+\beta=p+1>$ 2. Then for each fixed $b<0$ we have $d(b, c)=O\left(c^{\frac{p+3-2 \beta}{2(p-1)}}\right)$ as c approaches zero.

Proof. For $b<0$ and $0<c<b^{2} / 4$, let

$$
\lambda_{1}=\sqrt{\frac{-b-\sqrt{b^{2}-4 c}}{2}} \quad \lambda_{2}=\sqrt{\frac{-b+\sqrt{b^{2}-4 c}}{2}}
$$

Then $0<\lambda_{1}<\lambda_{2}$, and $e^{ \pm \lambda_{1} x}$ and $e^{ \pm \lambda_{2} x}$ are solutions of the linear equation $y^{\prime \prime \prime \prime}+b y^{\prime \prime}+c y=0$. Consider the trial function

$$
\psi(x)=\left\{\begin{array}{cc}
\lambda_{2} e^{-\lambda_{1} x}-\lambda_{1} e^{-\lambda_{2} x} & x \geq 0 \\
\lambda_{2} e^{\lambda_{1} x}-\lambda_{1} e^{\lambda_{2} x} & x<0
\end{array}\right.
$$


It is not difficult to check that $\psi$ is $C^{2}$, and decays exponentially to zero, and therefore $\psi \in H^{2}$. We now calculate

$$
\begin{aligned}
K(\psi) & =2 \int_{0}^{\infty}\left(\lambda_{2} e^{-\lambda_{1} x}-\lambda_{1} e^{-\lambda_{2} x}\right)^{\alpha} \lambda_{1}^{\beta} \lambda_{2}^{\beta}\left(e^{-\lambda_{1} x}-e^{-\lambda_{2} x}\right)^{\beta} d x \\
& \geq 2 \lambda_{1}^{\beta} \lambda_{2}^{\beta} \int_{0}^{\infty}\left(\lambda_{2} e^{-\lambda_{1} x}-\lambda_{1} e^{-\lambda_{1} x}\right)^{\alpha}\left(e^{-\lambda_{1} x}-e^{-\lambda_{2} x}\right)^{\beta} d x \\
& \geq 2 \lambda_{1}^{\beta} \lambda_{2}^{\beta}\left(\lambda_{2}-\lambda_{1}\right)^{\alpha} \int_{\frac{\log 2}{\lambda_{2}-\lambda_{1}}}^{\infty} e^{-\lambda_{1} \alpha x}\left(\frac{1}{2}\right)^{\beta} e^{-\lambda_{1} \beta x} d x \\
& =\lambda_{1}^{\beta-1}\left[\frac{\left(\frac{1}{2}\right)^{\beta-1} \lambda_{2}^{\beta}\left(\lambda_{2}-\lambda_{1}\right)^{\alpha} 2^{-\lambda_{1}(\alpha+\beta) /\left(\lambda_{2}-\lambda_{1}\right)}}{\alpha+\beta}\right]
\end{aligned}
$$

Since $\lambda_{1}=O(\sqrt{c})$ and $\lambda_{2} \rightarrow \sqrt{-b}$ as $c \rightarrow 0$, the bracketed term approaches

$$
\frac{\left(\frac{1}{2}\right)^{\beta-1}(\sqrt{-b})^{\beta+1}}{\alpha+\beta}>0
$$

as $c \rightarrow 0$. Thus since

$$
I(\psi)=\left(\lambda_{2}-\lambda_{1}\right) \lambda_{1} \lambda_{2}\left(\lambda_{1}^{2}-\lambda_{2}^{2}\right)=O(\sqrt{c})
$$

for $c$ near zero, we have

$$
m(b, c) \leq \frac{I(\psi)}{K(\psi)^{2 / p+1}}=O\left(c^{\frac{1}{2}-\frac{\beta-1}{p+1}}\right)=O\left(c^{\frac{p+3-2 \beta}{2(p+1)}}\right)
$$

and the lemma follows by equation (3.1).

Corollary 3.7. Let $F\left(u, u_{x}\right)=|u|^{\alpha}\left|u_{x}\right|^{\beta}$ or $F\left(u, u_{x}\right)=\operatorname{sign}(u)|u|^{\alpha}\left|u_{x}\right|^{\beta}$, where $\alpha+\beta=$ $p+1>2$, and $p+2 \beta<5$. Then for each $b<0$ there exist intervals arbitrarily close to $c=0$ on which $d$ is convex in $c$. Hence there exist c arbitrarily close to zero for which $\mathcal{G}(b, c)$ is stable.

Proof. This follows from the previous lemma since $\frac{p+3-2 \beta}{2(p-1)}>1$ when $p+2 \beta<5$, and since $d(b, c) \geq 0$ for all $c$. $b^{2} / 4$.

For the even nonlinearities, the same method gives a bound on $d$ for $b>0$ and $c$ near

Lemma 3.8. Let $F\left(u, u_{x}\right)=|u|^{\alpha}\left|u_{x}\right|^{\beta}$, where $\alpha+\beta=p+1>2$. Then for each fixed $b>0$ we have $d(b, c)=O\left(\left(c-b^{2} / 4\right)^{\frac{p+3}{2(p-1)}}\right)$ as c approaches $b^{2} / 4$.

Proof. For $c>b^{2} / 4$, the roots of $\lambda^{4}+b \lambda^{2}+c=0$ are $\lambda= \pm(\sigma \pm i \tau)$, where

$$
\sigma=\frac{1}{2} \sqrt{2 \sqrt{c}-b}, \quad \tau=\frac{1}{2} \sqrt{2 \sqrt{c}+b}
$$

Let

$$
\psi(x)=\left\{\begin{array}{cc}
e^{-\sigma x}(\tau \cos (\tau x)+\sigma \sin (\tau x)) & x \geq 0 \\
e^{\sigma x}(\tau \cos (\tau x)-\sigma \sin (\tau x)) & x \leq 0
\end{array}\right.
$$


The $\psi \in C^{2}$ and $\psi(x) \rightarrow 0$ as $|x| \rightarrow \infty$, so $\psi \in H^{2}$. Now $\psi$ is a solution of $\psi^{\prime \prime \prime \prime}+b \psi^{\prime \prime}+c \psi=0$ on $(-\infty, 0)$ and on $(0, \infty)$, so we have

$$
\begin{aligned}
I(\psi) & =2 f(0) \psi^{\prime \prime \prime}(0)+2 \int_{0}^{\infty} \psi\left(\psi^{\prime \prime \prime \prime}+b \psi^{\prime \prime}+c \psi\right) d x=2 \psi(0) \psi^{\prime \prime \prime \prime}(0) \\
& =4 \sigma \tau^{2}\left(\sigma^{2}+\tau^{2}\right)=\sqrt{c} \cdot \sqrt{c-b^{2} / 4} \cdot \sqrt{2 \sqrt{c}+b}
\end{aligned}
$$

for $\sigma$ near zero. Now

$$
\begin{aligned}
K(\psi) & =2\left(\sigma^{2}+\tau^{2}\right)^{\beta} \int_{0}^{\infty} e^{-\sigma(\alpha+\beta) x}|\tau \cos (\tau x)+\sigma \sin (\tau x)|^{\alpha}|\sin (\tau x)|^{\beta} d x \\
& \geq 2\left(\sigma^{2}+\tau^{2}\right)^{\beta} \sum_{n=0}^{\infty} \int_{\pi / 6 \tau+2 n \pi / \tau}^{\pi / 3 \tau+2 n \pi / \tau} e^{-\sigma(\alpha+\beta) x}\left(\frac{1}{2} \tau\right)^{\alpha}\left(\frac{1}{2}\right)^{\beta} d x \\
& \geq 2 c^{\beta / 2}\left(\frac{1}{2} \tau\right)^{\alpha}\left(\frac{1}{2}\right)^{\beta} \sum_{n=0}^{\infty} \int_{\pi / 6 \tau+2 n \pi / \tau}^{\pi / 3 \tau+2 n \pi / \tau} e^{-\sigma(\alpha+\beta) x} d x \\
& =\left[2 c^{\beta / 2}\left(\frac{1}{2} \tau\right)^{\alpha}\left(\frac{1}{2}\right)^{\beta} \frac{e^{-\frac{\sigma}{\tau}(\alpha+\beta) \pi / 6}-e^{-\frac{\sigma}{\tau}(\alpha+\beta) \pi / 3}}{\left(1-e^{-\frac{\sigma}{\tau}(\alpha+\beta) 2 \pi}\right)}\right] \sigma^{-1}
\end{aligned}
$$

Now as $c$ approaches $b^{2} / 4, \sigma$ approaches 0 and $\tau$ approaches $\frac{1}{2} \sqrt{2 b}$, so the bracketed term approaches

$$
\frac{b^{\beta+\alpha / 2}}{3 \cdot 2^{1+2 \beta+\frac{3}{2} \alpha}}>0
$$

Therefore, since $I(\psi)=O(\sigma)$ for $\sigma$ small, we have

$$
m(b, c) \leq \frac{I(\psi)}{K(\psi)^{2 / p+1}}=O\left(\sigma^{1+\frac{2}{p+1}}\right)=O\left(\sigma^{\frac{p+3}{p+1}}\right)=O\left(\left(c-b^{2} / 4\right)^{\frac{p+3}{2(p+1)}}\right)
$$

and it follows from $(3.1)$ that $d(b, c)=O\left(\left(c-b^{2} / 4\right)^{\frac{p+3}{2(p-1)}}\right)$.

Corollary 3.9. Let $F\left(u, u_{x}\right)=|u|^{\alpha}\left|u_{x}\right|^{\beta}$, where $\alpha+\beta=p+1>2$, and $p<5$. Then for each $b>0$ there exist intervals arbitrarily close to $c=b^{2} / 4$ on which $d$ is convex in $c$. Hence there exist $c$ arbitrarily close to $b^{2} / 4$ for which $\mathcal{G}(b, c)$ is stable.

Proof. This follows from the previous lemma since $\frac{p+3}{2(p-1)}>1$ when $p<5$, and since $d(b, c) \geq$ 0 for all $c$.

In light of our numerical results below, this result appears to be sharp, in the sense that for $p \geq 5$ (i.e. $\alpha+\beta \geq 6$ ) and $b>0$ we have $d^{\prime \prime}(c)<0$ for $c$ near $b^{2} / 4$. See Table 1 .

\section{Numerical Results}

Even with the scaling property of $d$, it is not possible to obtain an explicit formula for $d$ except in the case when $b=0$. However, by numerically approximating the solitary wave 
solutions, and using the fact that $d$ is given by (3.1), we can numerically approximate $d$. By doing this for several values of $c$ it is then possible to numerically approximate the second derivative of $d$.

In order to find the solitary waves, we use a one-parameter shooting method. For the nonlinearities considered here, the solitary wave equation (1.2) is reversible, so the solitary waves are even, and we assume that $\varphi_{x}(0)=\varphi_{x x x}(0)=0$. Multiplying (1.2) by $\varphi_{x}$ and integrating gives

$$
\varphi_{x x x} \varphi_{x}-\frac{1}{2} \varphi_{x x}^{2}+\frac{b}{2} \varphi_{x}^{2}+\frac{c}{2} \varphi^{2}=(1-\beta) F\left(\varphi, \varphi_{x}\right)
$$

where we have used the fact that $\varphi$ and its derivatives decay to zero as $|x| \rightarrow \infty$. We then use $a=\varphi(0)$ as the shooting parameter and $\varphi_{x x}(0)^{2}=c a^{2}-2(1-\beta) F(a, 0)$.

To test the accuracy of the computations, we compare the numerically computed solutions with the known exact formulas in the case $F(u)=u^{\alpha}$ and $\frac{b}{\sqrt{c}}=-\left(\frac{\alpha}{2}+\frac{2}{\alpha}\right)$. For example, when $\alpha=6, b=-1$ and $c=0.09$ we have the exact solution $\varphi(x)=\frac{1}{\sqrt{5}} \operatorname{sech}\left(\frac{x}{\sqrt{10}}\right)$. Figure 4 shows that the numerically computed solution is indistinguishable from the exact solution.

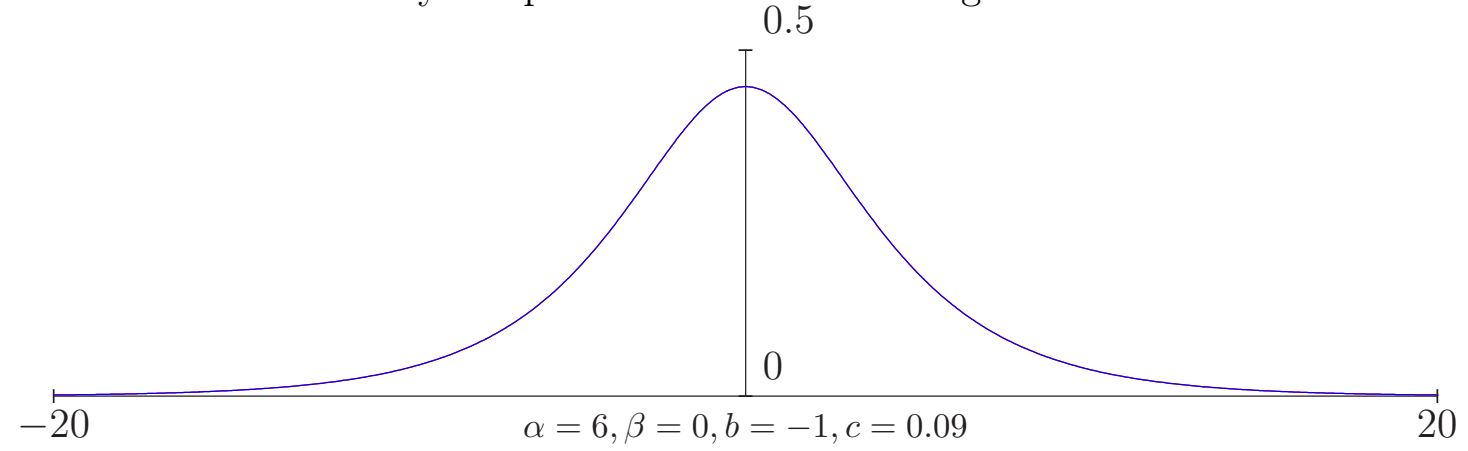

Figure 4: Plot of the exact solution together with the numerically computed solution.

Having found the solitary wave $\varphi$ with parameter values $(b, c)$ we then numerically integrate to find $K(\varphi)$ and apply equation (3.1) to find $d(b, c)$. Using Lemma 3.1, we also can compute its partial derivatives $d_{b}(b, c)$ and $d_{c}(b, c)$. We then do this for a collection of points $(b, c)$ along the segments $\Gamma_{1}$ and $\Gamma_{2}$ and use equations (3.4) and (3.5) to compute $d_{c c}$. For example, Figure 5 shows the computed values of $d_{c c}$ along $\Gamma_{1}$ and $\Gamma_{2}$ for the nonlinearity $F(u)=u^{7}$. The first plot indicates that $d_{c c}(-1, c)<0$ for $0<c<c_{0} \approx 0.114$. By the relation (3.5), this implies that $d_{c c}(b, c)<0$ for $(b, c)$ in the region $b / \sqrt{c}<b_{0} \approx-2.96$. The second plot indicates that $d_{c c}(b, 1)<0$ for $2>b>b_{1} \approx 1.69$. By equation (3.4), this implies that $d_{c c}(b, c)<0$ for $(b, c)$ in the region $b / \sqrt{c}>1.69$. In the region $-2.96<b / \sqrt{c}<1.69$ we have $d_{c c}(b, c)>0$. Thus by Theorem 1.2, solitary waves are stable in the region $-2.96<b / \sqrt{c}<1.69$ and unstable otherwise. The region of unstable solitary waves is the shaded region in Figure 6.

For the nonlinearities $F\left(u, u_{x}\right)=|u|^{\alpha}\left|u_{x}\right|^{\beta}$ and $F\left(u, u_{x}\right)= \pm \operatorname{sign}(u)|u|^{\alpha}\left|u_{x}\right|^{\beta}$ the results are summarized in Table 1 , and the figures that follow. The results are consistent with the analytical results of [11], [4] and [1]. More precisely, for $b=0$ we have stability when $p+2 \beta<9$ and instability when $p+2 \beta>9$. Note that $p+2 \beta=\alpha+3 \beta-1$, so the critical $(\alpha, \beta)$ pairs are $(10,0),(4,2)$ and $(1,3)$. For $p+2 \beta \geq 9$ we have instability for all $b<0$ and for $p+2 \beta>9$ we have instability for $b>0$ in some interval about zero, in agreement 

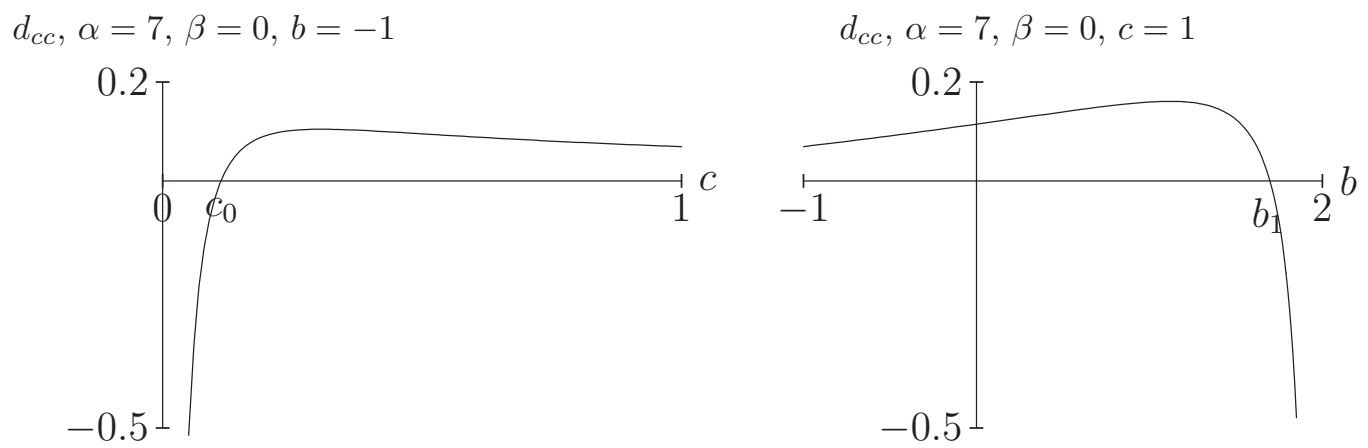

Figure 5: $d_{c c}$ along $\Gamma_{1}$ and $\Gamma_{2}$ for $\alpha=7, \beta=0$.

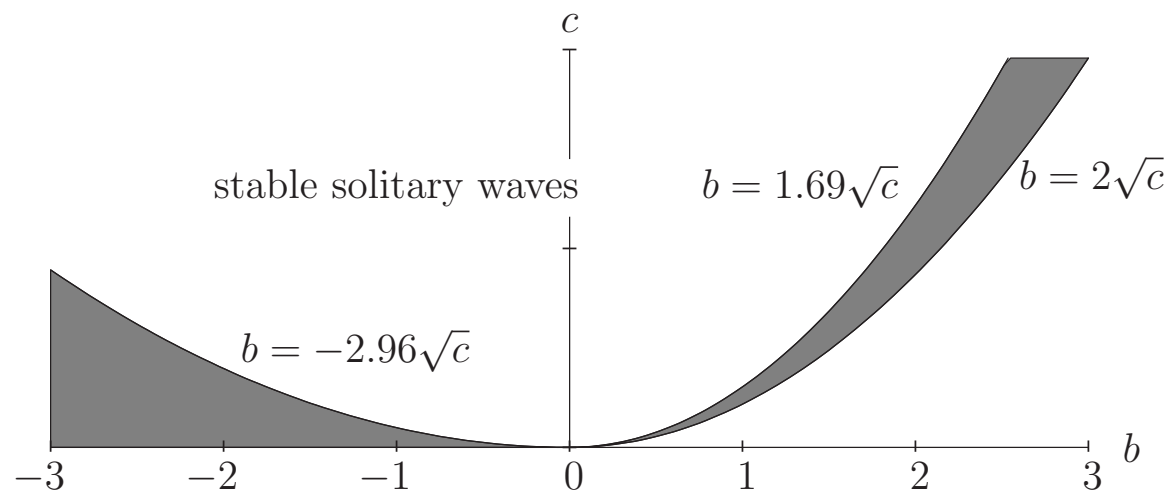

Figure 6: Stability region for $\alpha=7, \beta=0$.

with Theorem 1.3. These results extend those mentioned above in that they fairly precisely describe the entire regions of stable and unstable solitary waves in the $(b, c)$ parameter-plane. It is not clear how to obtain such results analytically. 


\begin{tabular}{|l|l|l|}
\hline \multicolumn{2}{|c|}{$F\left(u, u_{x}\right)=|u|^{\alpha}\left|u_{x}\right|^{\beta}$} \\
\hline \hline$\alpha$ & $\beta$ & Region of Stability \\
\hline 3 & 0 & $\frac{b}{\sqrt{c}}<2$ \\
\hline 4 & 0 & $\frac{b}{\sqrt{c}}<2$ \\
\hline 5 & 0 & $\frac{b}{\sqrt{c}}<1.806,1.892<\frac{b}{\sqrt{c}}<2$ \\
\hline 6 & 0 & $\frac{b}{\sqrt{c}}<1.744$ \\
\hline 7 & 0 & $-2.988<\frac{b}{\sqrt{c}}<1.677$ \\
\hline 8 & 0 & $-1.284<\frac{b}{\sqrt{c}}<1.602$ \\
\hline 9 & 0 & $-0.495<\frac{b}{\sqrt{c}}<1.521$ \\
\hline 10 & 0 & $0<\frac{b}{\sqrt{c}}<1.430$ \\
\hline 11 & 0 & $0.375<\frac{b}{\sqrt{c}}<1.319$ \\
\hline 12 & 0 & $0.745<\frac{b}{\sqrt{c}}<1.135$ \\
\hline 13 & 0 & none \\
\hline 14 & 0 & none \\
\hline 1 & 2 & $\frac{b}{\sqrt{c}}<2$ \\
\hline 2 & 2 & $-2.409<\frac{b}{\sqrt{c}}<2$ \\
\hline 3 & 2 & $-0.791<\frac{b}{\sqrt{c}}<1.760,1.861<\frac{b}{\sqrt{c}}<2$ \\
\hline 4 & 2 & $0<\frac{b}{\sqrt{c}}<1.632$ \\
\hline 5 & 2 & $0.522<\frac{b}{\sqrt{c}}<1.465$ \\
\hline 6 & 2 & none \\
\hline 7 & 2 & none \\
\hline 0 & 3 & $-0.992<\frac{b}{\sqrt{c}}<2$ \\
\hline 1 & 3 & $0<\frac{b}{\sqrt{c}}<2$ \\
\hline 2 & 3 & $0.596<\frac{b}{\sqrt{c}}<1.738,1.800<\frac{b}{\sqrt{c}}<2$ \\
\hline 3 & 3 & $1.199<\frac{b}{\sqrt{c}}<1.340$ \\
\hline 4 & 3 & none \\
\hline 5 & 3 & none \\
\hline 0 & 4 & $0.955<\frac{b}{\sqrt{c}}<2$ \\
\hline 1 & 4 & $1.553<\frac{b}{\sqrt{c}}<2$ \\
\hline 2 & 4 & none \\
\hline 3 & 4 & none \\
\hline 0 & 5 & $0.955<\frac{b}{\sqrt{c}}<2$ \\
\hline 1 & 5 & none \\
\hline 2 & 5 & none \\
\hline 0 & 6 & none \\
\hline 1 & 6 & none \\
\hline
\end{tabular}

\begin{tabular}{|c|l|l|}
\hline \multicolumn{3}{|c|}{$F\left(u, u_{x}\right)= \pm \operatorname{sign}(u)|u|^{\alpha}\left|u_{x}\right|^{\beta}$} \\
\hline \hline$\alpha$ & $\beta$ & Region of Stability \\
\hline 3 & 0 & $\frac{b}{\sqrt{c}}<2$ \\
\hline 4 & 0 & $\frac{b}{\sqrt{c}}<1.921$ \\
\hline 5 & 0 & $\frac{b}{\sqrt{c}}<1.870$ \\
\hline 6 & 0 & $\frac{b}{\sqrt{c}}<1.791$ \\
\hline 7 & 0 & $-2.988<\frac{b}{\sqrt{c}}<1.696$ \\
\hline 8 & 0 & $-1.284<\frac{b}{\sqrt{c}}<1.608$ \\
\hline 9 & 0 & $-0.495<\frac{b}{\sqrt{c}}<1.521$ \\
\hline 10 & 0 & $0<\frac{b}{\sqrt{c}}<1.430$ \\
\hline 11 & 0 & $0.375<\frac{b}{\sqrt{c}}<1.319$ \\
\hline 12 & 0 & $0.745<\frac{b}{\sqrt{c}}<1.135$ \\
\hline 13 & 0 & none \\
\hline 14 & 0 & none \\
\hline 1 & 2 & $\frac{b}{\sqrt{c}}<2$ \\
\hline 2 & 2 & $-2.409<\frac{b}{\sqrt{c}}<2$ \\
\hline 3 & 2 & $-0.791<\frac{b}{\sqrt{c}}<1.843$ \\
\hline 4 & 2 & $0<\frac{b}{\sqrt{c}}<1.695$ \\
\hline 5 & 2 & $0.522<\frac{b}{\sqrt{c}}<1.480$ \\
\hline 6 & 2 & none \\
\hline 7 & 2 & none \\
\hline 1 & 3 & $0<\frac{b}{\sqrt{c}}<1.907$ \\
\hline 2 & 3 & $0.595<\frac{b}{\sqrt{c}}<1.823$ \\
\hline 3 & 3 & $1.155<\frac{b}{\sqrt{c}}<1.480$ \\
\hline 4 & 3 & none \\
\hline 5 & 3 & none \\
\hline 1 & 4 & $1.419<\frac{b}{\sqrt{c}}<1.773$ \\
\hline 2 & 4 & none \\
\hline 3 & 4 & none \\
\hline 1 & 5 & none \\
\hline 2 & 5 & none \\
\hline 1 & 6 & none \\
\hline 2 & 6 & none \\
\hline
\end{tabular}

Table 1: Regions of Stability for $F\left(u, u_{x}\right)=|u|^{\alpha}\left|u_{x}\right|^{\beta}$ and $F\left(u, u_{x}\right)= \pm \operatorname{sign}(u)|u|^{\alpha}\left|u_{x}\right|^{\beta}$. 

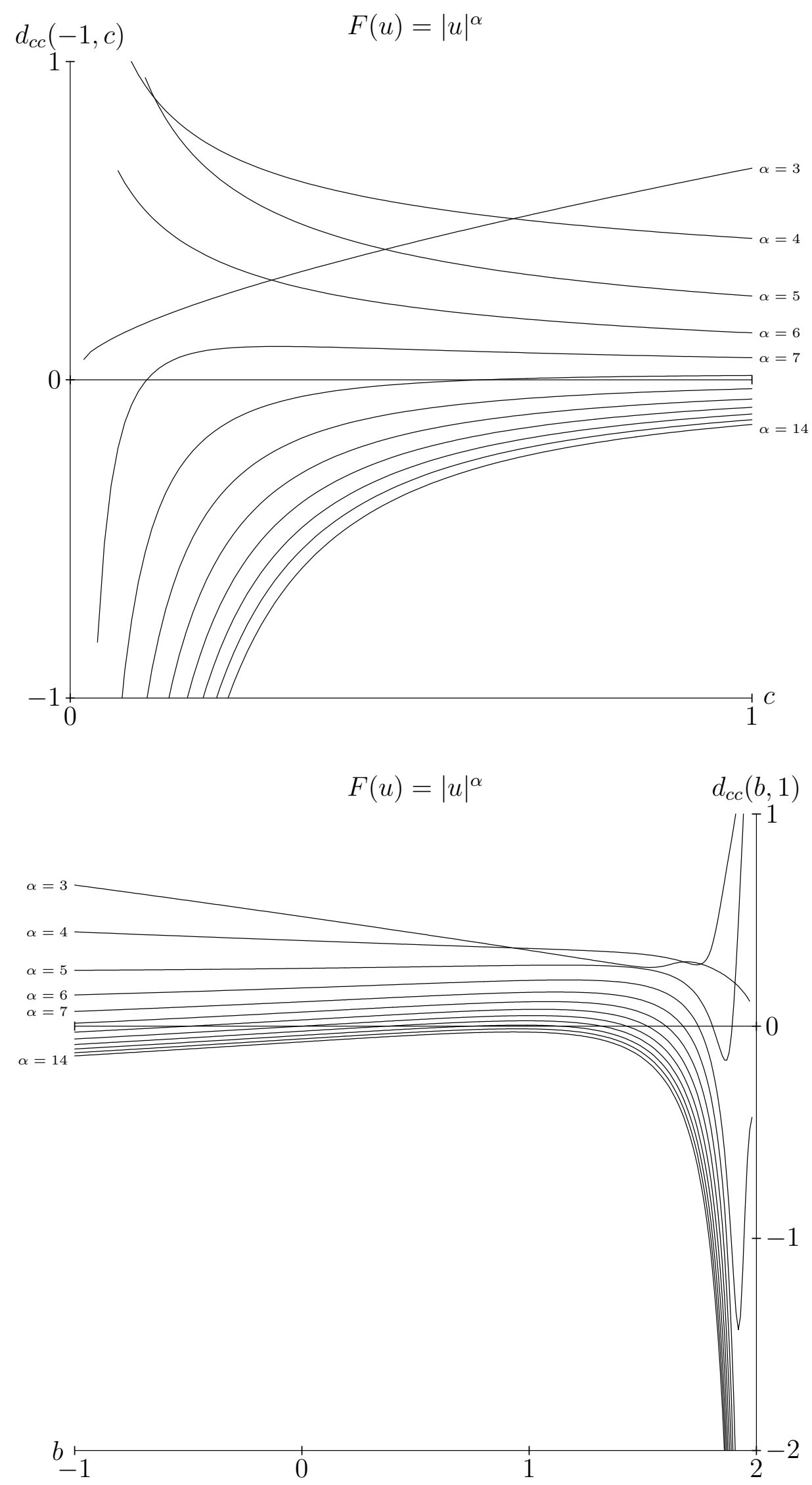

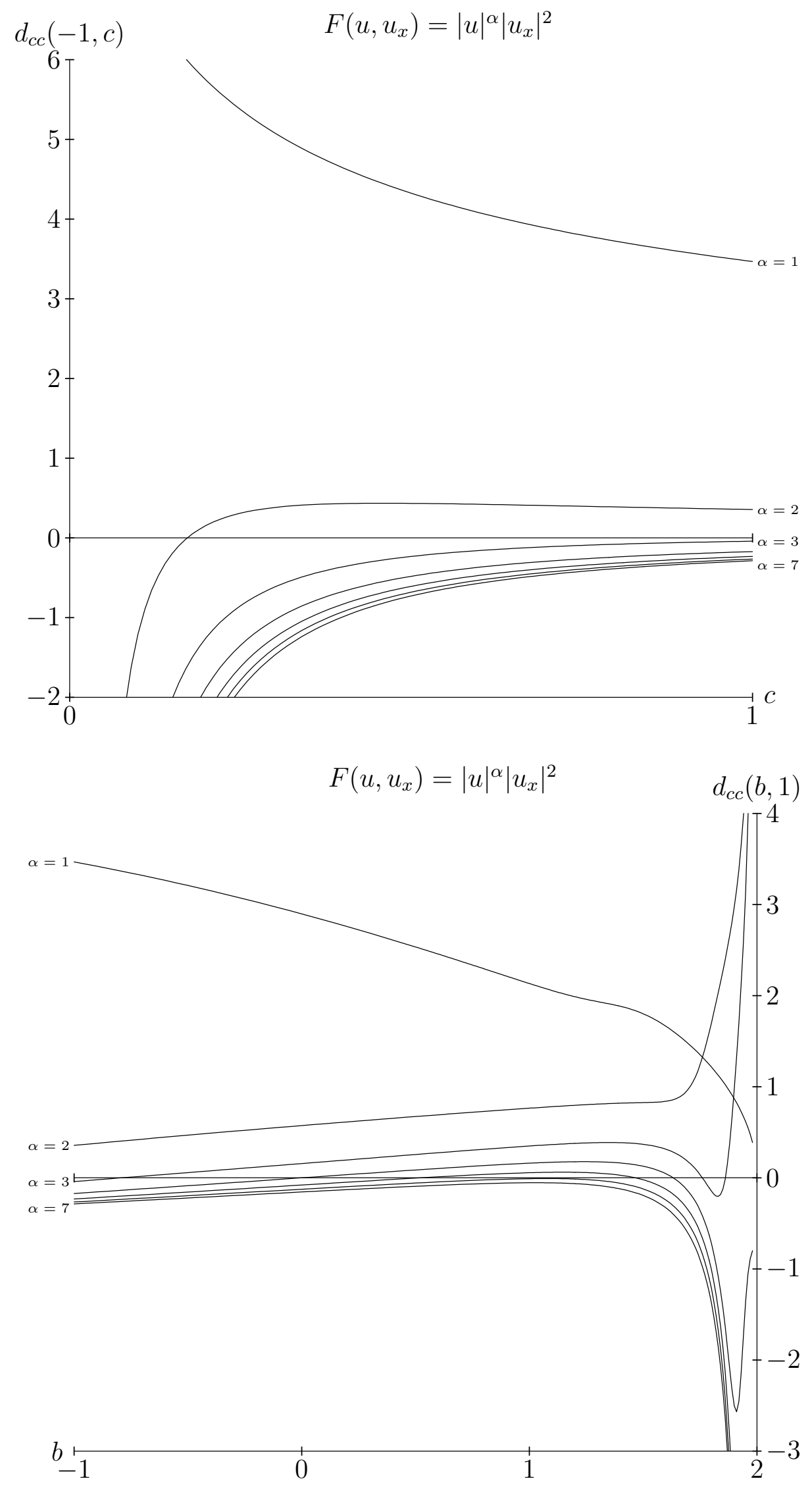

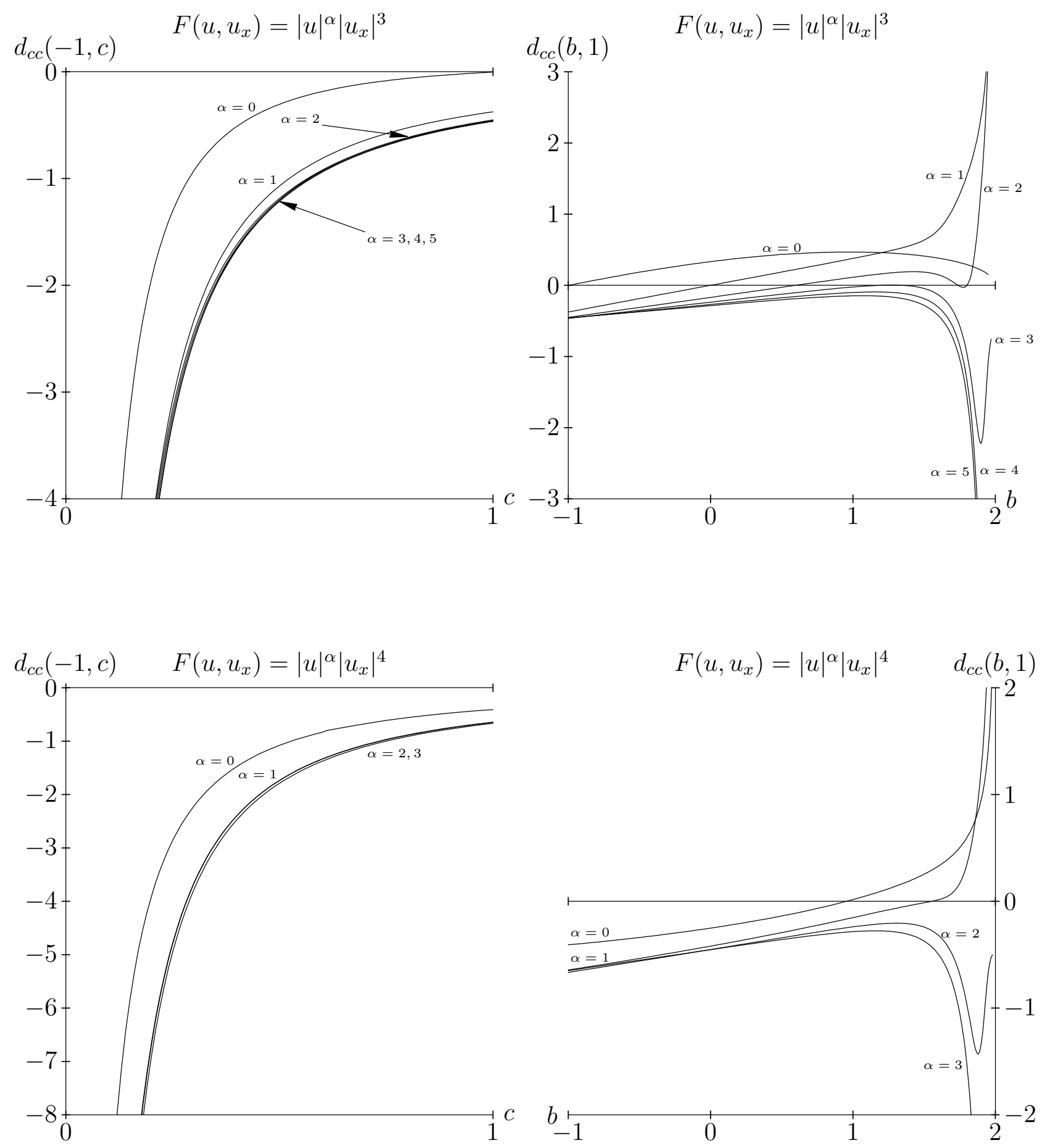

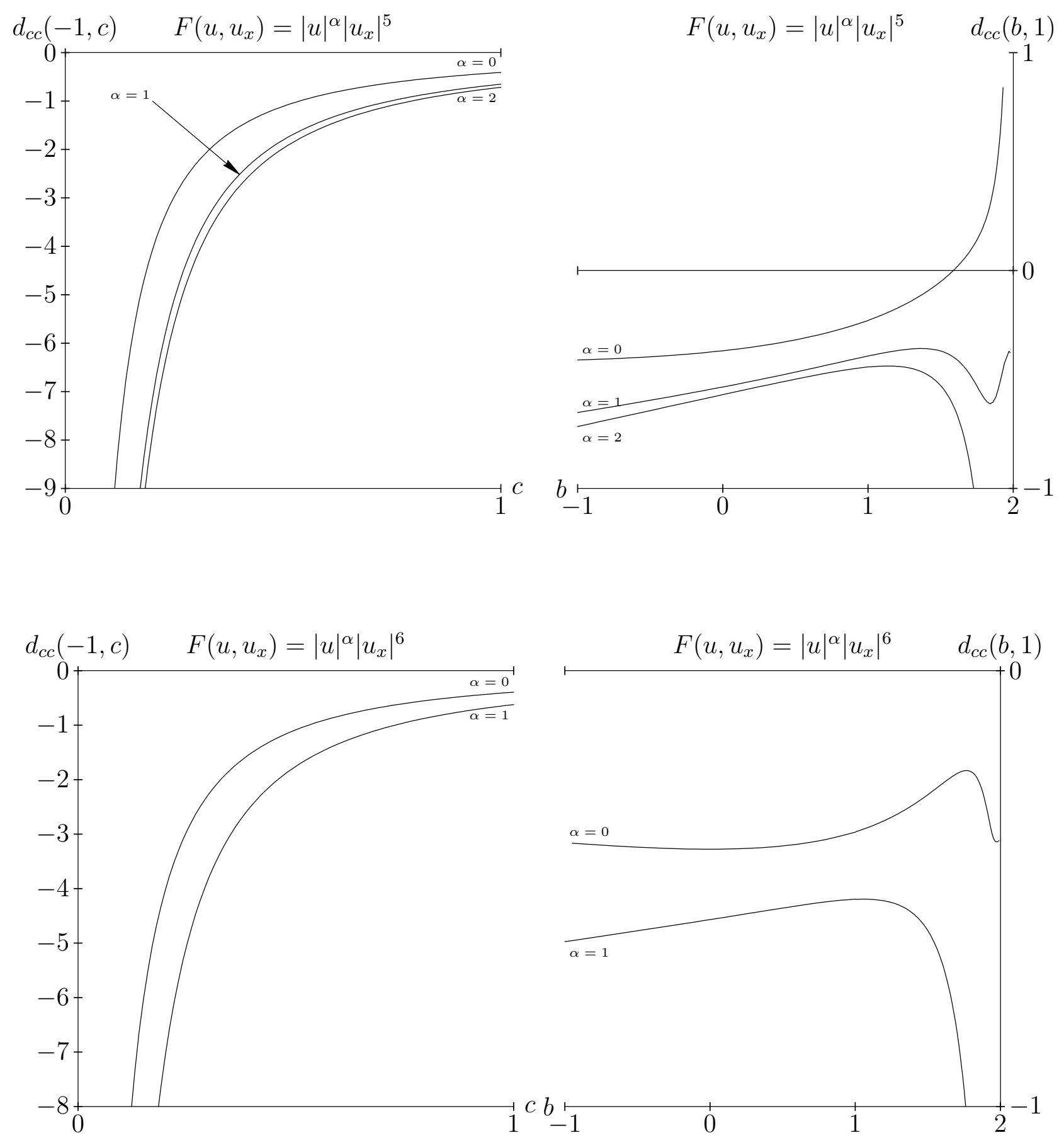

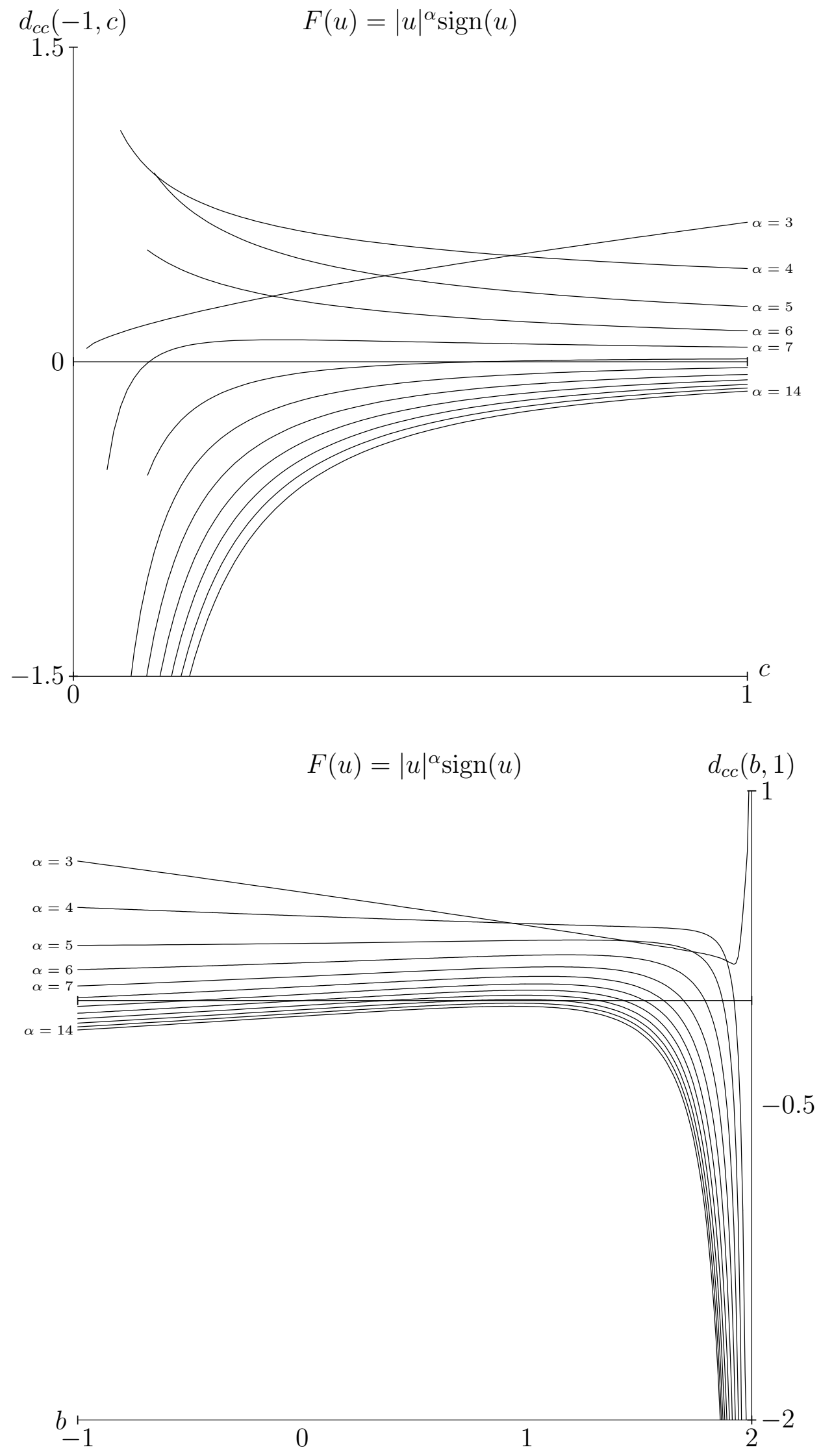

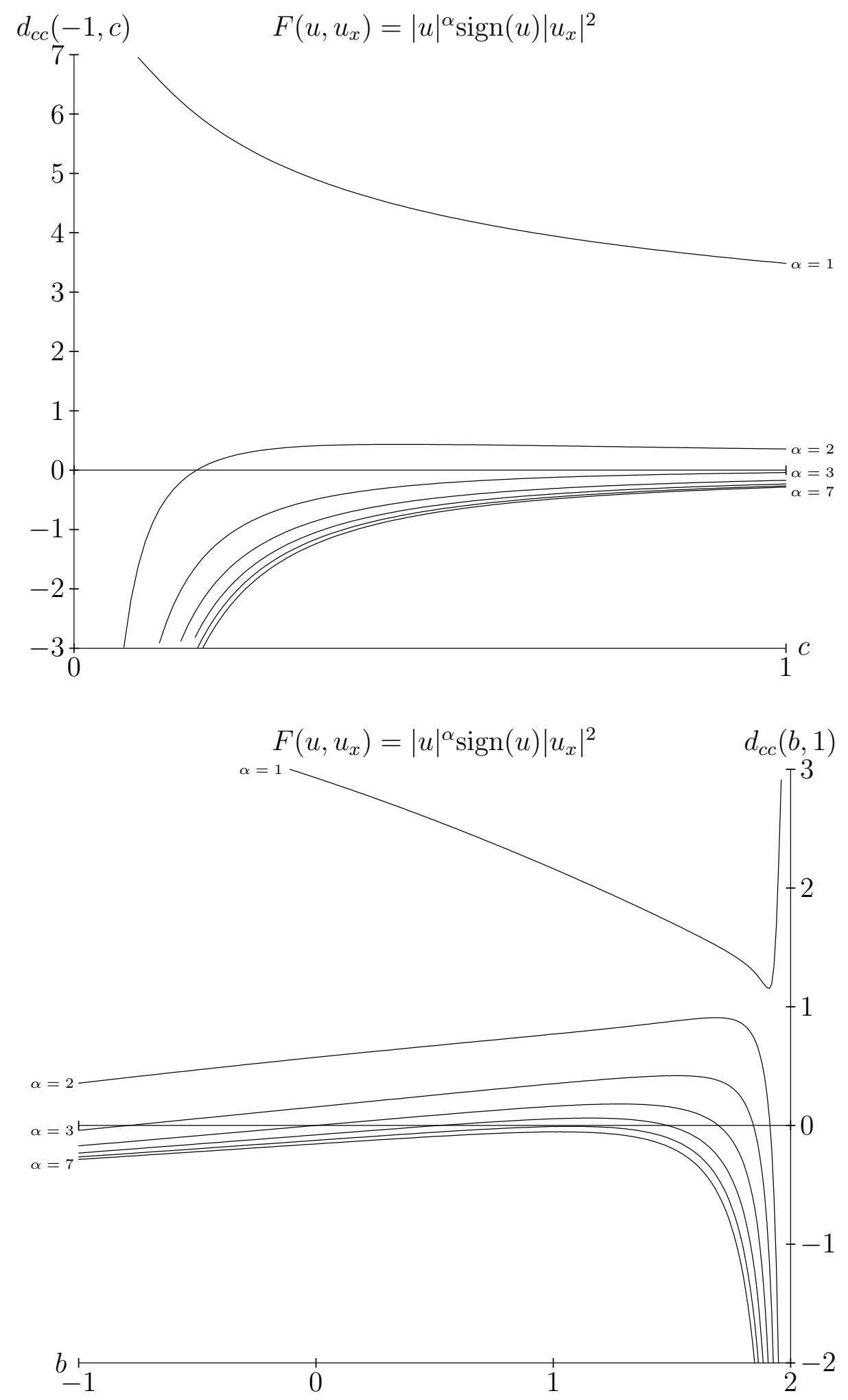

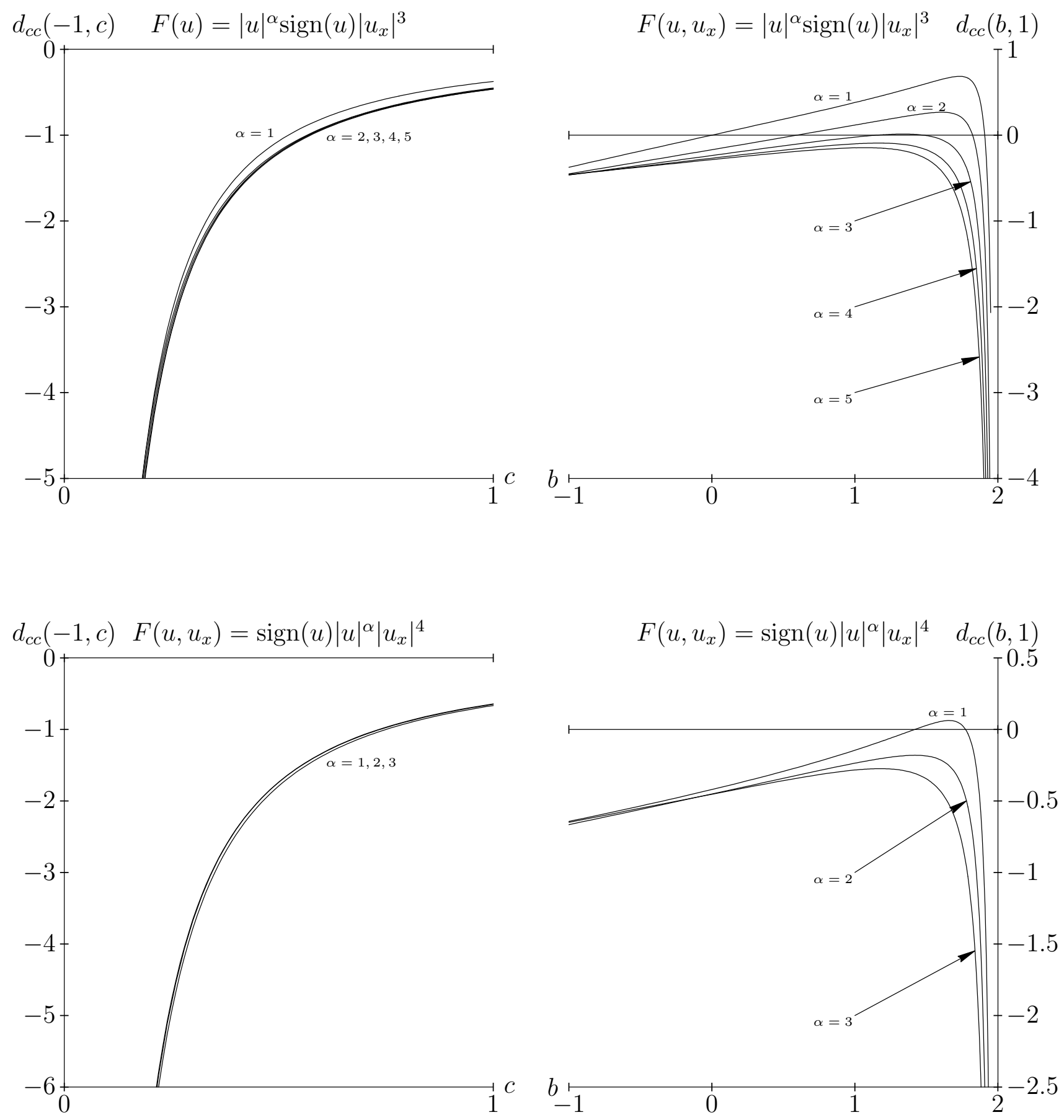

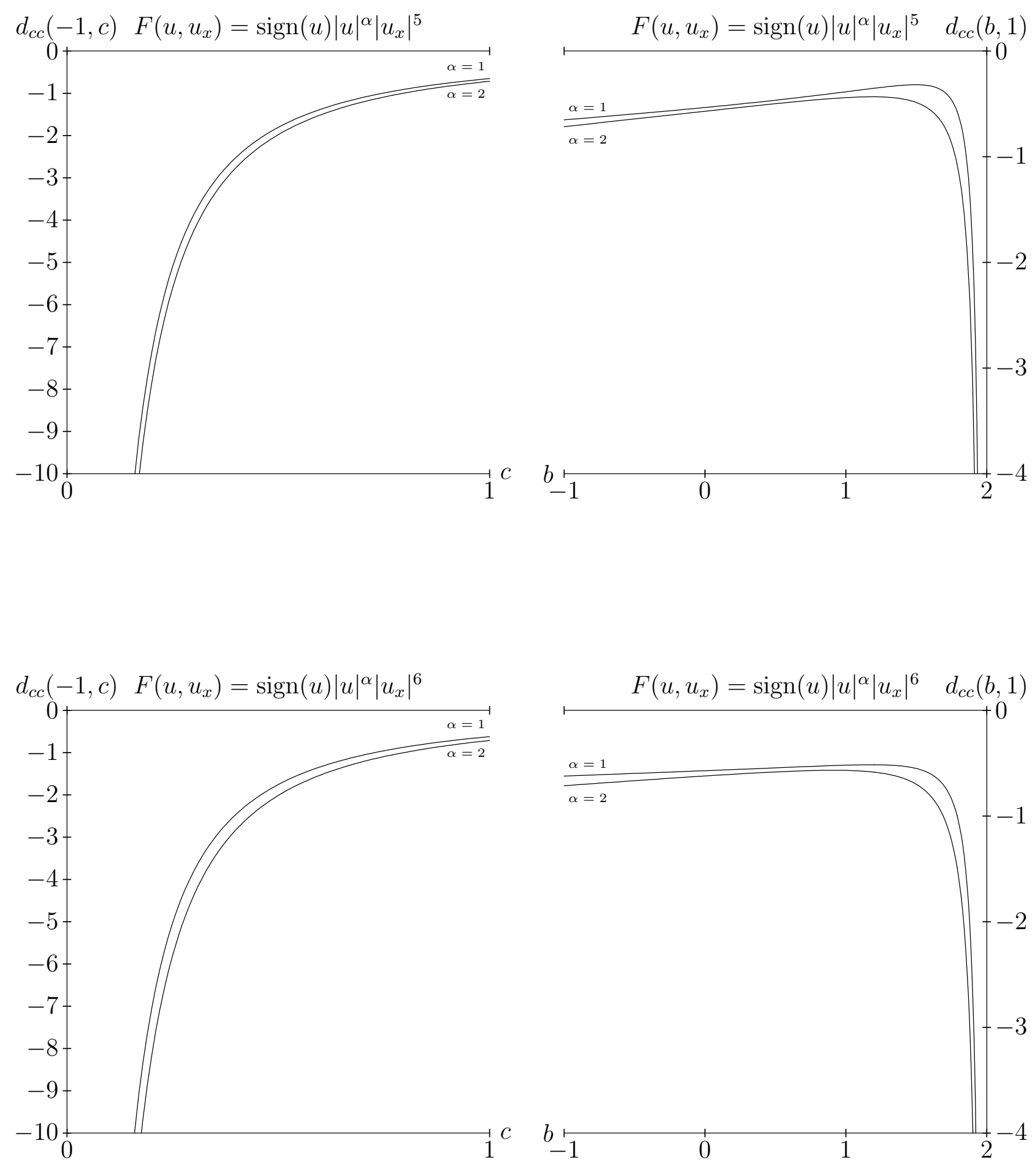


\section{References}

[1] J. Angulo Pava, On the instability of solitary-wave solutions for fifth-order water wave models. Electron. J. Differential Equations (2003), No. 6

[2] D.J. Benney, A general theory for interactions between short and long waves. Stud. Appl. Math. 56 (1977), 81-94

[3] J. Bona, P. Souganidis And W. Strauss, Stability and instability of solitary waves of Korteweg-de Vries type. Proc. Royal Soc. London Ser. A 411 (1987), 395-412.

[4] T. Bridges, G. Derks, Linear instability of solitary wave solutions of the Kawahara equation and its generalizations. SIAM J. Math. Anal. 33 (2002), No 6., 1356-1378

[5] T. Bridges, G. Derks and G. Gottwald, Stability and instability of solitary waves of the fifth-order KdV equation: a numerical framework. Phys. D 172 (2002), no. $1-4,190-216$.

[6] W. Craig and M.D. Groves, Hamiltonian long-wave approximations to the waterwave problem. Wave Motion 19 (1994), 367-389

[7] B. Dey, A. Khare and C. N. Kumar, Stationary solitons of the fifth order KdVtype equations and their stabilization. Phys. Lett. A, 223 (1996), no. 6, 449-452

[8] J. M. Gonçalves Ribeiro, Instability of symmetric stationary states for some nonlinear Schrödinger equations with an external magnetic field. Ann. Inst. H. Poincaré; Phys. Théor. 54 (1992), 403-433.

[9] J. Hunter And J. Scheurle, Existence of perturbed solitary wave solutions to a model equation for water waves. Physica D 32 (1988), 253-268

[10] M. Grillakis, J. Shatah And W. Strauss, Stability theory of solitary waves in the presence of symmetry I. J. Funct. Anal. 74 (1987), no. 1, 160-197

[11] S. Levandosky, A stability analysis of fifth-order water wave models. Physica D, 125 (1999), 222-240.

[12] P.L. Lions, The concentration-compactness principle in the calculus of variations. The locally compact case. Part I and Part II. Ann. Inst. Henri Poincare'Sect A (N.S.) 1 (1984), 109-145, 223-283

[13] P.J. Olver, Hamiltonian and non-Hamiltonian models for water waves. Lecture Notes in Physics. No. 195, Springer Verlag. (1984) 273-290

[14] J.A. ZufiRIA, Symmetry breaking in periodic and solitary gravity-capillary waves on water of finite depth. J. Fluid Mech 184 (1987), 183-206 